\title{
Anomalous couplings in single top quark and searches for rare top quark couplings with the ATLAS detector
}

\author{
Susana Cabrera Urbán*† \\ Instituto de Física Corpuscular (IFIC) University of Valencia and CSIC, Valencia, Spain \\ E-mail: cabreralific.uv.es
}

\begin{abstract}
Two recent analyses that have exhaustively explored potential anomalous couplings in the Wtb vertex using $t$-channel single-top-quark events selected from collision data at $\sqrt{s}=8 \mathrm{TeV}$ with the ATLAS detector are reviewed. The first one measures the top-quark polarisation and six $W$-boson spin opservables from angular asymmetries unfolded to parton level. The second one measures the normalised triple-differential angular decay rate of top quarks to simultaneously constrain all the anomalous couplings. The limits on the anomalous couplings improve the existing limits set by ATLAS at $\sqrt{s}=7 \mathrm{TeV}$. In addition, searches for flavour-changing neutral current top-quark interactions based on data collected by ATLAS and CMS, are reviewed. Finally, a recent search for $t \rightarrow q H$, with $H \rightarrow \gamma \gamma$, performed by ATLAS at $\sqrt{s}=13 \mathrm{TeV}$ is also presented, where previous LHC limits are improved.
\end{abstract}

The European Physical Society conference on High Energy Physics

5-12 July 2017

Venice, Italy

${ }^{*}$ Speaker.

${ }^{\dagger}$ On behalf of the ATLAS collaboration. 


\section{Introduction}

The top quark is the heaviest known fundamental particle and probing its couplings with the other fundamental particles may open a window to physics beyond the Standard Model (SM). At the LHC, in proton-proton collisions, the dominant production of top quarks is in pairs $(t \bar{t})$ via the flavour-conserving strong interaction while the single production of top quarks happens via charged-current electroweak processes involving a $W t b$ vertex. The $t$-channel is the dominant production mechanism of single top quarks, and it allows to test the coupling between the top quark, the $W$ boson and the $b$ quark, since it involves the $W t b$ vertex in at both the top quark production and decay. Moreover, in the $t$-channel, top quarks are produced with a large degree of polarisation along the direction of the produced light-flavour quark, called the spectator quark [1], thereby making $t$-channel events the ideal probe to measure the top-quark polarisation and $W$ boson spin observables. The effective Lagrangian describing the $W t b$ vertex can be expressed in terms of complex anomalous couplings $V_{\mathrm{L}, \mathrm{R}}$ and $g_{\mathrm{L}, \mathrm{R}}$ following the equation:

$$
\mathscr{L}_{W t b}=-\frac{g}{\sqrt{2}} \bar{b} \gamma^{\mu}\left(V_{\mathrm{L}} P_{\mathrm{L}}+V_{\mathrm{R}} P_{\mathrm{R}}\right) t W_{\mu}^{-}-\frac{g}{\sqrt{2}} \bar{b} \frac{i \sigma^{\mu v} q_{v}}{m_{W}}\left(g_{\mathrm{L}} P_{\mathrm{L}}+g_{\mathrm{R}} P_{\mathrm{R}}\right) t W_{\mu}^{-}+\text {h.c. }
$$

In the SM at leading order, $V_{\mathrm{L}}=\left|V_{\mathrm{tb}}\right| \approx 1$ and $V_{\mathrm{R}}=g_{\mathrm{L}}=g_{\mathrm{R}}=0$. Deviations from these values would indicate physics beyond the SM. Moreover, complex values of these couplings would imply that the top-quark decay has a CP-violating component [2]. On the other hand, searches for rare topquark decays are interesting because a wide variety of models of physics beyong the SM predicts a strong enhancement of the branching ratios of flavor-violating top quark decays [3], that are highly suppressed in the SM.

\section{Anomalous couplings in the $W t b$ vertex using $t$-channel single-top-quark production}

The two analyses reviewed $[4,5]$ have used $20.2 \mathrm{fb}^{-1}$ of data collected at $8 \mathrm{TeV}$ with the ATLAS detector [6] at the LHC. very similar event selections, background nomalisations and modelling. The preselection criteria of the $t$-channel experimental signature starts with one isolated lepton, electron or muon, with $E_{\mathrm{T}}>25 \mathrm{GeV}$, exactly two jets with $E_{\mathrm{T}}>30 \mathrm{GeV}$, only one jet $b$ tagged and missing transverse momentum, $E_{\mathrm{T}}^{\text {miss }}$, greater that $30 \mathrm{GeV}$. After some requirements to ensure a proper reconstruction quality of the event, the transverse mass of the lepton- $E_{\mathrm{T}}^{\text {miss }}$ system is required to be greater than $50 \mathrm{GeV}$ and a requirement on events having the lepton and the highest$p_{\mathrm{T}}$ jet with opposite directions in the transverse plane is applied to reduce the multijet background. Events with an additional lepton identified with less stringent criteria and with $p_{T}>10 \mathrm{GeV}$ are rejected to reduce backgrounds with two leptons, mainly dileptonic $t \bar{t}$. A further discrimination between the $t$-channel signal events and background events is achieved by applying four additional requirements: $\left|\eta_{\text {non b-jet }}\right|>2, \Delta\left(\eta_{\text {non b-jet }}, \eta_{\mathrm{b}-\text { jet }}\right)>1.2$, the mass of the reconstructed top quark between $130 \mathrm{GeV}$ and $200 \mathrm{GeV}$ and $H_{\mathrm{T}}>195 \mathrm{GeV}$. ${ }^{1}$

The background modelling is checked in two background-dominated regions defined right after the preselection criteria exclusing the veto of events with two leptons. A region enriched in $t \bar{t}$ events is defined with two additional untagged jets. A second region enriched in $W+$ jets

\footnotetext{
${ }^{1} H_{\mathrm{T}}$ is the scalar sum of the $p_{\mathrm{T}}$ of the lepton, the $p_{\mathrm{T}}$ of the jets and the $E_{\mathrm{T}}^{\text {miss }}$.
} 
events is selected with a loose $b$-tagged jet and vetoing jets that have been $b$-tagged as in the signal region. An additional region, enriched in $W+$ jets events and called "anti-signal", is built with preselection requirements and failing any of the four signal selection requirements. This region has a flavour composition similar to the one in the signal region and thus it is appropiated to constrain the $W+$ jets normalisation. The signal and the background event yields are determined through a maximum-likelihood fit to the number of observed data events in the signal, "anti-signal" and $t \bar{t}$ enriched regions. The shapes of signal and background are based on Monte Carlo (MC), except for the multijet background, determined using a data-driven matrix method. After the normalisation fit, the signal to background ratio is $\sim 1.18$, and the relative background composition is $40 \%$ of $t \bar{t}$, $42 \%$ of $W+$ jets and a remaining $18 \%$ of minor backgrounds: $W t$ and $s$-channel single-top-quark production, $\mathrm{Z}+$ jets, diboson production and multijet events.

The first analysis [4] has measured the top-quark polarisation $\mathrm{P}$ and the six independent $W$ boson spin observables proposed in Refs. [2, 7]: $\left\langle S_{1,2,3}\right\rangle,\left\langle T_{0}\right\rangle$ and $\left\langle A_{1,2}\right\rangle$. The observables $\left\langle S_{1,2}\right\rangle$ and $\left\langle A_{1,2}\right\rangle$ are proportional to $\mathrm{P}$. The observables $\left\langle S_{3}\right\rangle$ and $\left\langle T_{0}\right\rangle$ are related the $W$-boson helicity fractions: $F_{\mathrm{R}}, F_{\mathrm{L}}$ and $F_{0} \cdot{ }^{2}$ All these observables and $\mathrm{P}$ are extracted from eight different angular asymmetries, unfolded to parton level, and compare with theoretical predictions. In particular, $A_{\mathrm{FB}}^{\mathrm{N}}=-\frac{3}{4}\left\langle S_{2}\right\rangle$, the forward-backward asymmetry in the angular distribution $\cos \theta_{\ell}^{\mathrm{N}} 3$ is very sensitive to $\operatorname{Im} g_{\mathrm{R}}$ (see Figure 1(a)) and the forward-backward asymmetry in the angular distribution $\cos \theta_{\ell}{ }^{4}, A_{\mathrm{FB}}^{\ell}$, is used to measure the top-quark polarisation (see Figure 1(b)).

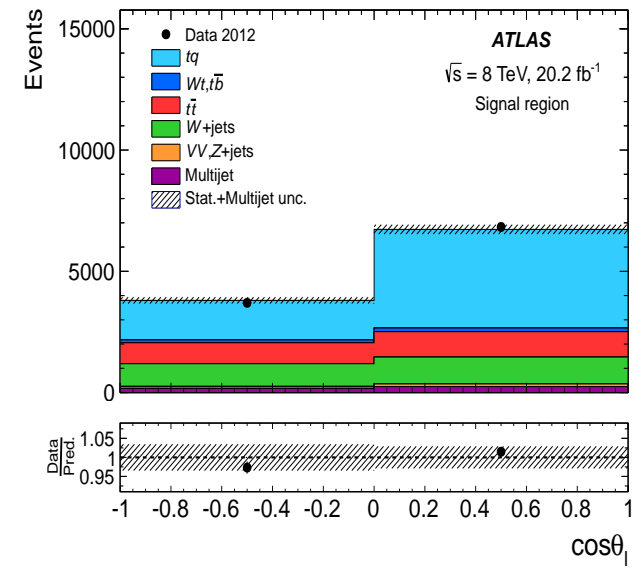

(a)

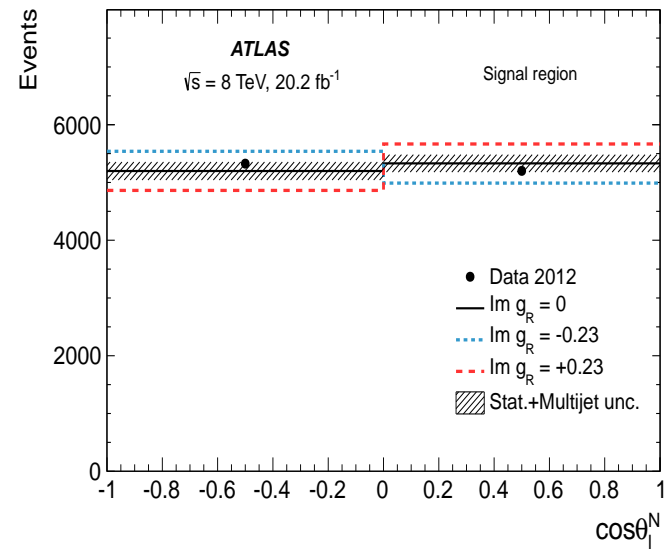

(b)

Figure 1: Angular distribution of $\cos \theta_{\ell}$ in the signal region (a) [4] and comparison of the measured distribution of $\cos \theta_{\ell}^{\mathrm{N}}$ with various theoretical predictions assuming different $\operatorname{Im} g_{\mathrm{R}}$ values (b) [4].

To unfold the eight angular asymmetries, except $A_{\mathrm{FB}}^{\mathrm{N}}$, the unfolding corrections (i.e, migration matrix and selection efficiencies) are determined with Monte Carlo samples of $t$-channel events produced with PROTOS [8] generator with SM values of the $W t b$ couplings. In the case of $A_{\mathrm{FB}}^{\mathrm{N}}$,

\footnotetext{
${ }^{2}$ The longitudinal and transverse (lelf- and right-handed) $W$-boson helicity fractions are $F_{0}, F_{\mathrm{L}}$ and $F_{\mathrm{R}}$, respectively.

${ }^{3}$ The angle $\theta_{\ell}^{\mathrm{N}}$ is the relative angle between the lepton momentum in the $W$-boson rest frame and the normal axis to the plane defined by the top-quark spin direction, taken along the spectator-quark momentum in the top-quark rest frame, and the $W$-boson momentumn the top-quark rest frame.

${ }^{4}$ The angle $\theta_{\ell}$ is the angle between the lepton momentum in the top-quark rest frame and the top-quark spin axis.
} 
an iterative method with Lagrange interpolation is used to determine the unfolding corrections using MC simulation samples generated with РRотоS with non zero values of $\operatorname{Im} g_{\mathrm{R}}$. For all the eight asymmetries measured, the systematic uncertainties are larger than the statistical ones, being the dominant sources of systematics the modelling of $t \bar{t}$ events, the jet energy scale and the MC statistics.

The measurements of the six $W$-boson spin observables are compatible with the SM predictions with an overall $p$-value of 0.83 (see Figure 2(a)). The observables $\left\langle S_{1,}\right\rangle$ and $\left\langle A_{1,2}\right\rangle$ are measured for the first time. From $A_{\mathrm{FB}}^{\ell}$ and $A_{\mathrm{FB}}^{\mathrm{N}}$, measured independently on any assumption on $\operatorname{Im}\left\{g_{R}\right\}$ and assuming $V_{\mathrm{L}}=1$ and $V_{\mathrm{R}}=g_{\mathrm{L}}=\operatorname{Re} g_{\mathrm{R}}=0$, the limits placed on $\operatorname{Im} g_{\mathrm{R}}$ at the $95 \%$ confidence level (CL) are: $\operatorname{Im} g_{\mathrm{R}} \in[-0.18,0.06]$. These limits improve the previous limits set by ATLAS at 7 $\mathrm{TeV}$ [11]. The measured value of the product of the top-quark polarisation and the charged-lepton spin analysing power extracted from $A_{\mathrm{FB}}^{\ell}: \alpha_{\ell} P=0.97 \pm 0.05$ (stat.) \pm 0.11 (syst.) is in agreement with the theoretical predictions at NLO $[9,10]$.

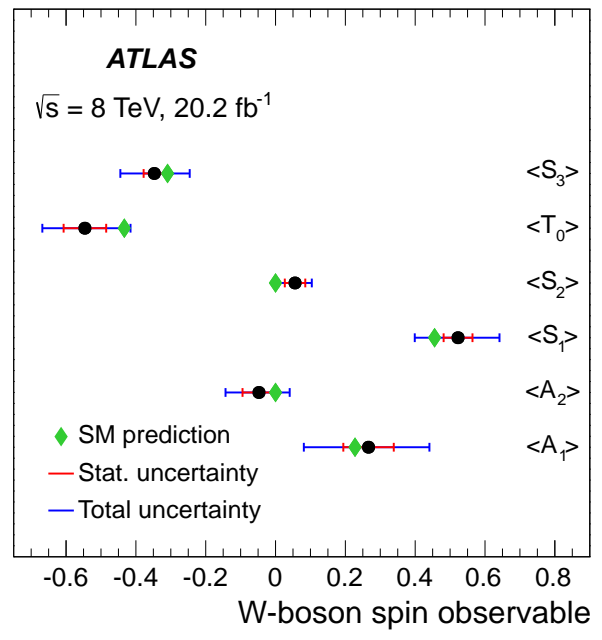

(a)

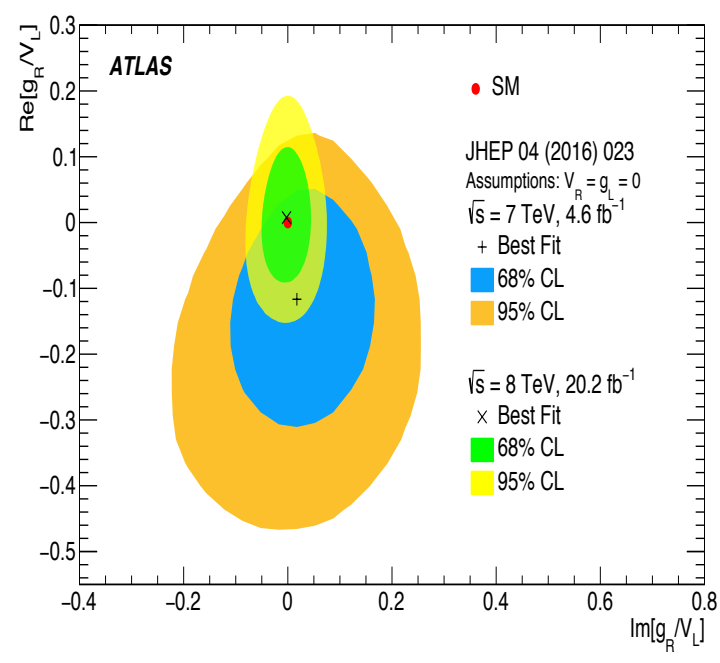

(b)

Figure 2: Summary of the measured six $W$-boson spin observables compared with the SM predictions (a) [4]. Contour limits on complex values of the ratio of anomalous couplings $g_{\mathrm{R}} / V_{\mathrm{L}}$ (b) [5].

The second analysis of the $W t b$ vertex carried out by the ATLAS Collaboration [11] is based on the measurement of the triple-differential angular decay rate of single top quarks produced in $t$-channel events to simultaneously determine five generalised helicity fractions and phases together with the top-quark polarisation from which the anomalous couplings are constrained. The moments of the measured angular distribution are estimated using an orthonormal series density estimation technique. A multidimensional likelihood function is built, where detector effects are deconvolved from data using a Fourier technique. From numerical calculations of this likelihood function, contour limits as the one shown in Figure 2(b) are obtained and limits are placed simultaneously on possible complex values of ratios of the anomalous couplings with no assumptions on values of the other anomalous couplings: $\left|V_{\mathrm{R}} / V_{\mathrm{L}}\right|<0.37,\left|g_{\mathrm{L}} / V_{\mathrm{L}}\right|<0.29, \operatorname{Re}\left[g_{\mathrm{R}} / V_{\mathrm{L}}\right] \in[-0.2,0.17]$ and $\operatorname{Im}\left[g_{\mathrm{R}} / V_{\mathrm{L}}\right] \in[-0.07,0.06]$ at $95 \% \mathrm{CL}$. These limits improve the previous ones from the double- 
differential angular analysis [5] performed by ATLAS at $\sqrt{s}=7 \mathrm{TeV}$.

\section{Searches for rare top quark decays}

The branching ratios (BRs) of flavor-changing neutral curremts (FCNC) top quark decay, are highly suppressed in the SM due to the Glashow-Iliopoulos-Maiani (GIM) mechanism. However, various new physics models predict a strong enhancement in these BRs [3]. For instance, models with two Higgs-boson doublets with flavour violating Yukawa couplings, 2HDM(FV), predict $\mathrm{BR}(t \rightarrow H c)=2 \times 10^{-3}$ and $\mathrm{BR}(t \rightarrow g c) \leq 10^{-4}$. The existing limits placed by ATLAS and CMS at $\sqrt{s}=8 \mathrm{TeV}$ are summarized in Figure 3(a). It is worth to mention that the closest upper limits at $\sqrt{s}=8 \mathrm{TeV}$ to the theoretical expectations predicted by new physics models are: $\mathrm{BR}(t \rightarrow H c)<4.0 \times 10^{-3}[12]$ and $\mathrm{BR}(t \rightarrow g c)<2 \times 10^{-4}[13]$.

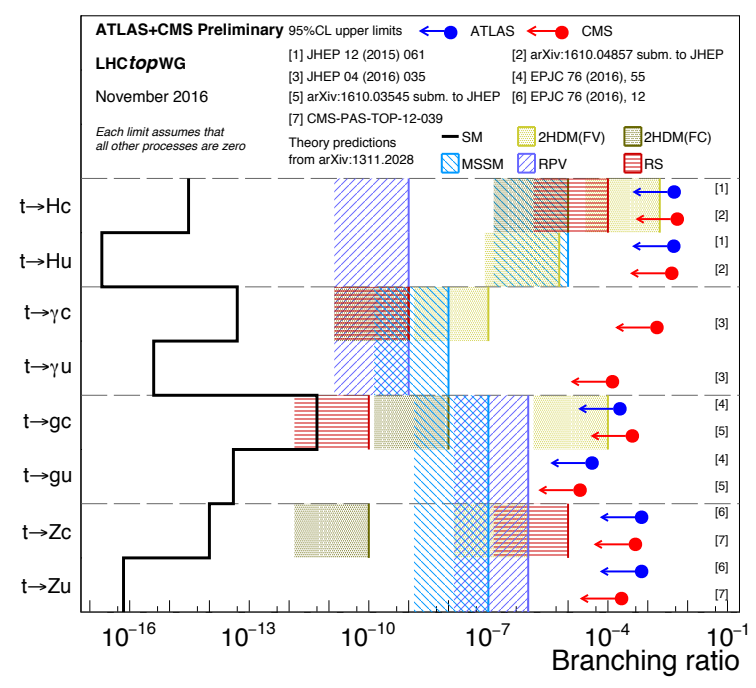

(a)

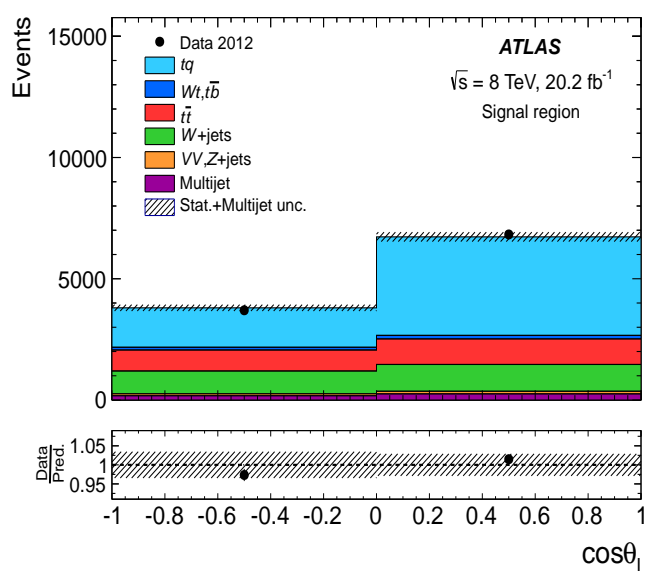

(b)

Figure 3: Latest summary of top-quark FCNC BRs from the LHCtopWG (a) [14]. Diphoton invariant mass distribution for the best subcathegory in the hadronic mode (b) [15].

A new search for top-quark decays $t \rightarrow q H$, with $H \rightarrow \gamma \gamma$, has been performed by the ATLAS Collaboration at $\sqrt{s}=13 \mathrm{TeV}$ [15]. Two modes are included in the analysis, the hadronic mode: $t \bar{t} \rightarrow W\left(\rightarrow q q^{\prime}\right) b+H(\rightarrow \gamma \gamma) b$ and the leptonic mode: $t \bar{t} \rightarrow W(\rightarrow \ell v) b+H(\rightarrow \gamma \gamma) b$. Besides selecting two photons, four jets and at least one $b$-tagged jet are required in the hadronic mode, and one lepton (electron or muon) with $p_{\mathrm{T}}>10 \mathrm{GeV}$ and at least two jets in the leptonic one. In addition, two or one combinations of invariant masses kinematically compatible with two top quark decays are required in both modes, defining two subcathegories. The main backgrounds are the non-resonant diphoton production plus one jet $\gamma \gamma j, t \bar{t} \gamma \gamma,(W+Z) \gamma \gamma$ and $t t H(\rightarrow \gamma \gamma)$. The diphoton invariant mass $m_{\gamma \gamma}$ of signal and background is fitted to data (see Figure 3(b)). No significant excess is observed and an observed (expected) upper limit is set on $\mathrm{BR}(t \rightarrow c H)$ branching ratio of $2.2(1.6) \times 10^{-3}$ at the $95 \% \mathrm{CL}$, tantalizingly close to the predictions from $2 \mathrm{HDM}(\mathrm{FV})$ models.

\section{Conclusions}

The ATLAS Collaboration has exhaustively probed the $W t b$ vertex structure using $t$-channel single-top-quark production at $\sqrt{s}=8 \mathrm{TeV}$. The eight measured asymmetries and six $W$ boson spin 
observables together with the top-quark polarisation are in agreement with the SM predictions. A novel interpolation technique has allowed to constrain $\operatorname{Im} g_{\mathrm{R}}$ assuming Standard Model values for the rest of the couplings. The measurement of triple-differential angular decay rates has allowed to simultaneously extract limits on complex values of ratios of the anomalous couplings with no assumptions on values of the other anomalous couplings. A recent search for FCNC interactions in top-quark decays $t \rightarrow q H$ performed with ATLAS at $\sqrt{s}=13 \mathrm{TeV}$ has improved previous LHC limits, entering in the region of sensitivity to 2HDM with flavor violating Yukawa couplings.

\section{Acknowledgements}

S.C. is indebted to all the colleagues, funding agencies, CERN and institutions that are contributing to the amazing effort of the LHC Physics program. The work of S.C. is funded by the project FPA2015-65652-C4-1-R of the Spanish Ministry of Economy, Industry and Competitiveness and the project Severo Ochoa Center of Excellence of IFIC: SEV-2014-0398.

\section{References}

[1] G.Mahlon and S.Parke, Improved Spin Basis for Angular Correlation Studies in Single Top Quark Production at the Tevatron, Phys.Rev.D 55 (1997) 7249

[2] J.A Aguilar Saavedra and J.Bernabéu, W polarization beyond helicity fractions in top quark decays, Nucl.Phys.B 840 (2010) 349

[3] K.Agashe et al., Snowmass 2013 Top quark working group report, arXiv: 1311.2028 [ hep-ph] .

[4] ATLAS Collaboration, Probing the Wtb vertex structure in t-channel single-top-quark production and decay in pp collisions at $\sqrt{s}=8 \mathrm{TeV}$ with the ATLAS detector, JHEP 04 (2017) 124

[5] ATLAS Collaboration, Analysis of the Wtb vertex from the measurement of triple-differential angular decay rates of single top quarks produced in the $t$-channel at $\sqrt{s}=8 \mathrm{TeV}$ with the ATLAS detector, arXiv: $1707.05393[$ hep-ex].

[6] ATLAS Collaboration, The ATLAS Experiment at the CERN Large Hadron Collider, JINST 3 (2008) S08003

[7] J.A Aguilar Saavedra and J.Bernabéu, Breaking down the entire W boson spin observables from its decay, Phys.Rev.D 93 (2016) 011301

[8] J.A Aguilar Saavedra, Single top quark production at LHC with anomalous Wtb couplings, Nucl.Phys.B 804 (2008) 160

[9] R. Schwienhorst, C.P. Yuan, C. Mueller and Q.-H. Cao, Single top quark production and decay in the t-channel at next-to-leading order at the LHC, Phys.Rev.D 83 (2011) 034019

[10] A. Brandenburg, Z.G. Si and P. Uwer, QCD corrected spin analyzing power of jets in decays of polarized top quarks, Phys.Lett.B 539 (2002) 235

[11] ATLAS Collaboration, Search for anomalous couplings in the Wtb vertex from the measurement of double differential angular decay rates of single top quarks produced in the t-channel with the ATLAS detector, JHEP 04 (2016) 023

[12] CMS Collaboration, Search for top quark decays via Higgs-boson-mediated flavor-changing neutral currents in pp collisions at $\sqrt{s}=8 \mathrm{TeV}$, JHEP 1702 (2017) 079

[13] ATLAS Collaboration, Search for single top-quark production via flavour-changing neutral currents at $8 \mathrm{TeV}$ with the ATLAS detector, Eur.Phys.J. C76 (2016) no.2, 55 
[14] https://twiki.cern.ch/twiki/bin/view/LHCPhysics/LHCTopwGSummaryPlots

[15] ATLAS Collaboration, Search for top quark decays $t \rightarrow q H$, with $H \rightarrow \gamma \gamma$, in $\sqrt{s}=13$ TeV pp collisions using the ATLAS detector, arXiv:1707.01404 [hep-ex]. 